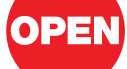

SUBJECT AREAS:

PHENOLOGY

ECOSYSTEM ECOLOGY

ANIMAL PHYSIOLOGY

FOOD WEBS

Received

20 December 2012

Accepted

25 April 2013

Published

9 May 2013

Correspondence and requests for materials should be addressed to

L.M. (laura.

mckinnon3@gmail.

com)

\section{Arctic-nesting birds find physiological relief in the face of trophic constraints}

\author{
Laura McKinnon' ${ }^{1}$, Erica Nol' \& Cédric Juillet ${ }^{2}$
}

${ }^{1}$ Trent University, Department of Biology, 2140 East Bank Drive, Peterborough, ON, Canada, K9J 7B8, ${ }^{2}$ Trent University, Department of Environmental and Resource Studies/Science, 1600 West Bank Drive Peterborough, ON, Canada K9J 7B8.
A climate-induced phenological mismatch between the timing of reproduction and the timing of food resource peaks is one of the key hypothesized effects of climate change on wildlife. Though supported as a mechanism of population decline in birds, few studies have investigated whether the same temperature increases that drive this mismatch have the potential to decrease energetic costs of growth and compensate for the potential negative effects of reduced food availability. We generated independent indices of climate and resource availability and quantified their effects on growth of Dunlin (Calidris alpina) chicks, in the sub-arctic tundra of Churchill, Manitoba during the summers of 2010-2011 and found that when resource availability was below average, above average growth could be maintained in the presence of increasing temperatures. These results provide evidence that chicks may find physiological relief from the trophic constraints hypothesized by climate change studies.

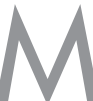
igratory systems such as those seen in arctic-nesting shorebirds, that travel thousands of kilometres from tropical and southern hemisphere non-breeding grounds to the Arctic and sub-Arctic tundra, presumably evolved, in part, to take advantage of the short-lived but abundant burst of food resources for reproduction ${ }^{1}$. Though abundant in resources, the tundra is by no means a warm and hospitable environment for the offspring of arctic-breeding birds. Daily energy expenditure of arctic-nesting shorebirds is $50 \%$ higher than that of temperate nesting birds and it is during reproduction that daily energy expenditure of shorebirds is the highest across the annual cycle ${ }^{2}$. Arctic-nesting shorebirds have evolved a host of adaptations to deal with reproduction in this cold and unpredictable environment such as a clutch size of 4 eggs that maximizes heat retention and incubation efficiency ${ }^{3}$, large egg sizes which optimize the size of chicks at hatch, and highly mobile precocial chicks with high metabolism ${ }^{2}$, and fast growth rates ${ }^{4,5}$. Fast growth rates of chicks are particularly important in the short arctic summer as chicks need to attain thermoregulatory independence rapidly to take advantage of the abundant foraging opportunities, and to build fat reserves for migration to their wintering grounds ${ }^{4,6}$.

In birds, reproduction should be timed such that clutches will hatch a few days prior to seasonal peaks in food resources permitting chicks to maximize the amount of food available for growth ${ }^{7}$. This synchrony appears to be so important that asynchrony between hatch and food peaks has recently been suggested as one of the key factors in the population declines of several bird species ${ }^{8-11}$. These population level effects of asynchrony between hatch and peaks in food resources in birds are thought to occur because food peaks on the breeding grounds are occurring earlier as temperatures increase, yet in some species of migratory birds, timing of breeding has either not changed, or not advanced enough to meet these peaks, leading to reduced food for offspring and decreases in growth and survival of young ${ }^{10}$. Although many of the pioneering studies highlighting mismatch as a mechanism of population decline focussed on temperate breeding altricial birds ${ }^{8,10}$, evidence of the same mechanisms of population declines in precocial birds is mounting ${ }^{9,12}$.

Arctic-nesting birds may be particularly vulnerable to a mismatch between hatch and food resource peaks due to the short arctic breeding season and climate driven peaks in food availability ${ }^{13,14}$ (but see ${ }^{15}$ ). Although growth rates of shorebird chicks may be hindered by asynchrony between hatch and food resource peaks, we hypothesized that the same temperature increases that potentially drive asynchrony may also decrease energetic requirements of chicks via reduced thermoregulatory costs. Up to one third of the total energy budget of young chicks is allocated to foraging and thermoregulation ${ }^{16}$, and greater thermoregulatory costs in the presence of low temperatures and rain may retard growth ${ }^{17,18}$. Under windy conditions, heat loss and metabolic rate may increase by up to $50 \%$ in arctic-nesting chicks with the greatest effect on younger chicks ${ }^{19}$. We hypothesized that temperature increases during the brood-rearing period have the potential to reduce thermoregulatory costs and compensate for a reduction in food resources due to a mismatch. 
To investigate whether any potential climate-driven mismatch with peaks in food abundance could be compensated by reduced thermoregulatory costs of chicks due to increased temperatures during the brood-rearing season, we generated independent indices of climate and insect availability and quantified their respective effects on chick growth. We monitored the growth of free living chicks of Dunlin (Calidris alpina, $n=106$ chicks from 35 families) during 2 breeding seasons (2010 and 2011) in the sub-arctic tundra of Churchill, Manitoba $\left(58^{\circ} 45^{\prime} \mathrm{N}, 94^{\circ} 04^{\prime} \mathrm{W}\right)$. To determine the relative importance of climate and food availability in explaining variation in growth, we generated indices of relative growth by extracting the growth residuals (observed - expected) from the best fitting growth model for three morphometric measures (mass, tarsus, culmen, Figure 1). We then constructed linear mixed-effects models to explain variation in our indices of growth based on factors related to climate and food availability (Supplementary Table S1). In order to deal with the lack of independence both within and between the set of climate and food availability covariates, we ran a principal components analysis on 10 covariates ( 5 climate and 5 food availability) in order to generate a set of synthetic orthogonal, or non-correlated, variables to represent climate and food availability ${ }^{20,21}$. Our main prediction was that both temperature and arthropod biomass would have positive effects on the growth residuals (Hypothesis 6, Supplementary Table S1), however, to support our hypothesis that increasing temperatures may have a compensatory effect, we predicted that a contour plot of growth residuals (plotted against temperature and arthropod indices) would show positive relief (indicating above average growth) at temperatures at and above the current mean, even when arthropod values were below the mean (Figure 2a). Given that shorebird chicks do not gain independence in

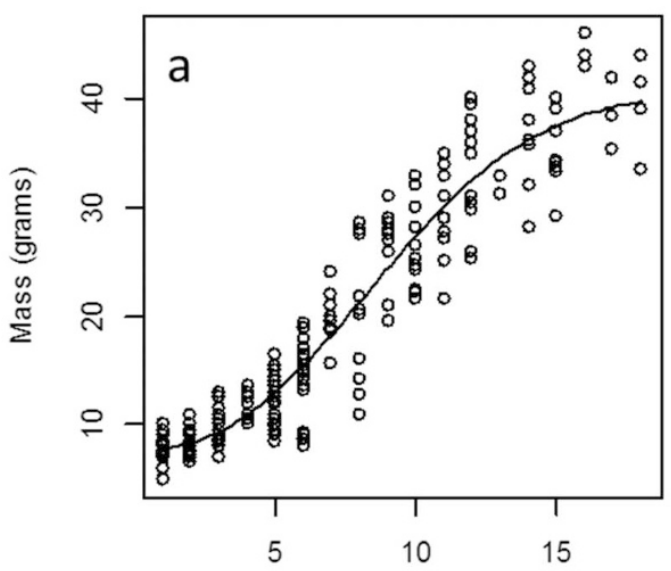

Age (days)

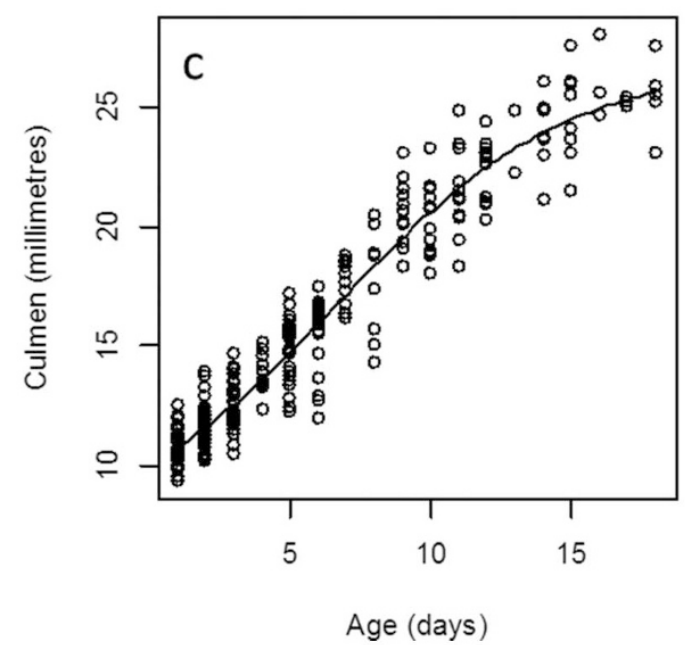

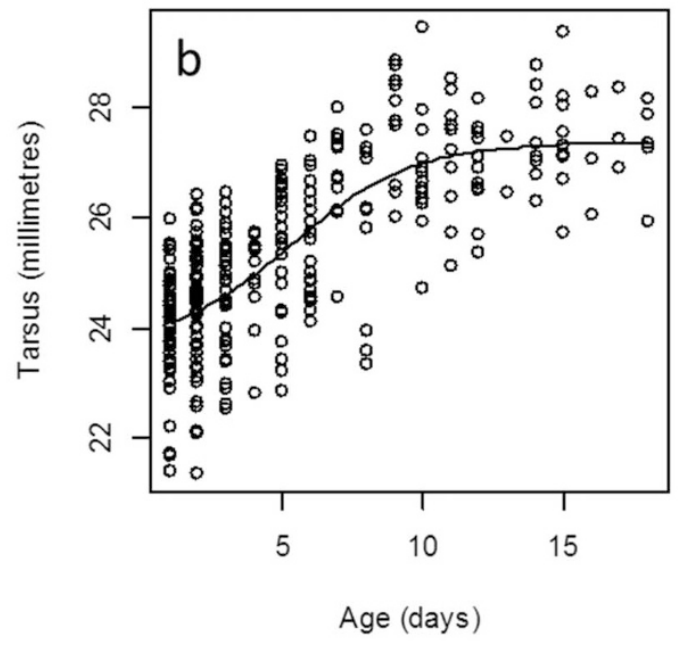

Age (days)

Figure $1 \mid$ Growth models for mass by age (a), tarsus by age (b), and culmen by age (c). Raw data points are presented along with the modeled growth curves. a) The 4-parameter Weibull Type 2 model was the best fitting growth model to describe mass as a function of age, though the 4-parameter $\log$-Logistic model was also competitive $(\triangle \mathrm{AIC}=0.14$; Supplementary Table $\mathrm{S} 4)$. Based on the best fitting model, the mean mass of chicks at hatch (age 1 ) was $7.71 \pm 0.32 \mathrm{~g}$ and increased to an average upper limit of $40.60 \pm 1.23 \mathrm{~g}$ at day 18 (Supplementary Table S5), 71\% of adult mean body mass ( $57.56 \pm 0.5 \mathrm{~g}$, range $47-68, \mathrm{n}=77$ ). b) Growth of the tarsus (diagonal) by age was best described by the 4 parameter logistic model, with the 4-parameter Weibull Type 2 ( $\triangle$ AIC 0.38; Supplementary Table S4) and the 4 parameter log-Logistic ( $\triangle$ AIC 1.23) also competitive. Based on the best fitting model, mean tarsus length of chicks at hatch (age 1) was $24.12 \pm 0.16 \mathrm{~mm}$ and grew to an average upper limit of $27.68 \pm 0.38 \mathrm{~mm}$ at day 18 (Supplementary Table S5), falling within the range of average adult tarsus length (27.62 \pm 0.17 , range 22.1-31.5, $\mathrm{n}=77)$. Tarsus length varied little from hatch to fledging $(\sim 4 \mathrm{~mm})$. c) The 4-parameter logistic model was also the best model to describe the growth of culmen by age (Supplementary Table S4), with the 4 parameter Weibull Type 2 and the 4 parameter log-Logistic also competitive ( $\triangle$ AIC 0.3 and 1.5 respectively). Mean culmen length of chicks at hatch (age 1) was $6.77 \pm 1.25 \mathrm{~mm}$, then increased to an upper limit of $26.84 \pm 0.76 \mathrm{~mm}$ at day 18 (Supplementary Table S5), approximately $74 \%$ of adult mean culmen length (36.37 $\pm 0.29 \mathrm{~mm}$, range $29.62-43.39, \mathrm{n}=77)$. 


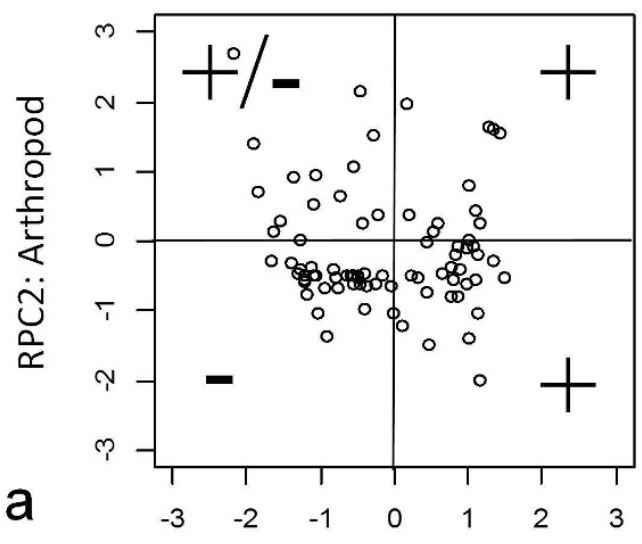

RPC1: Temperature

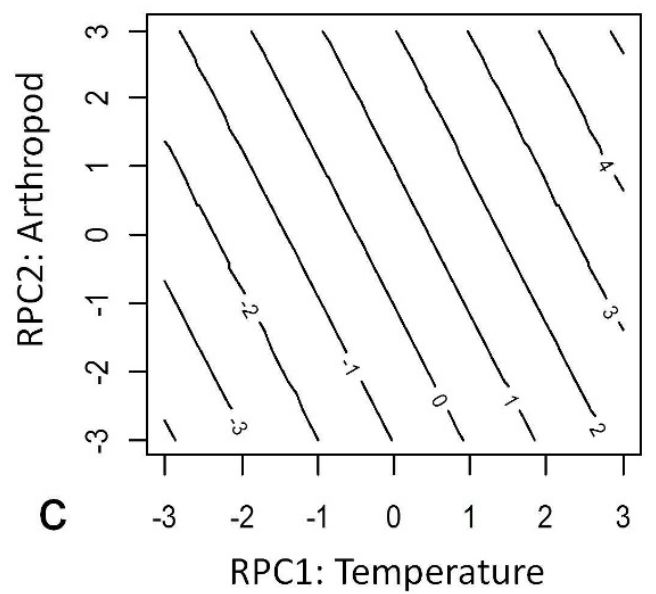

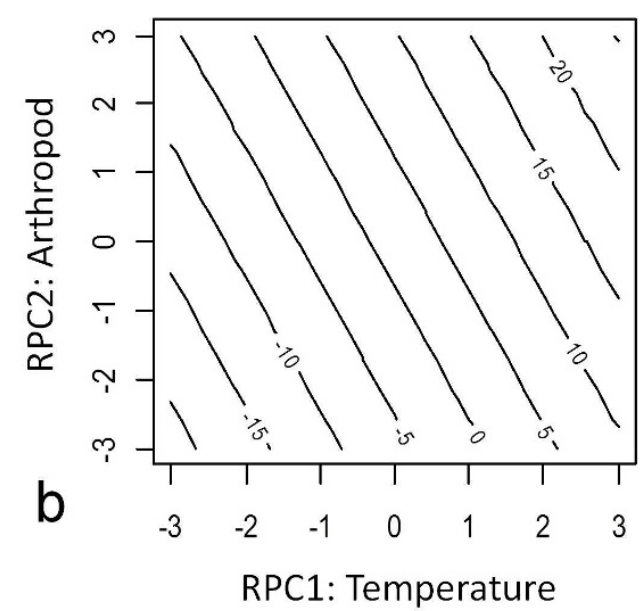

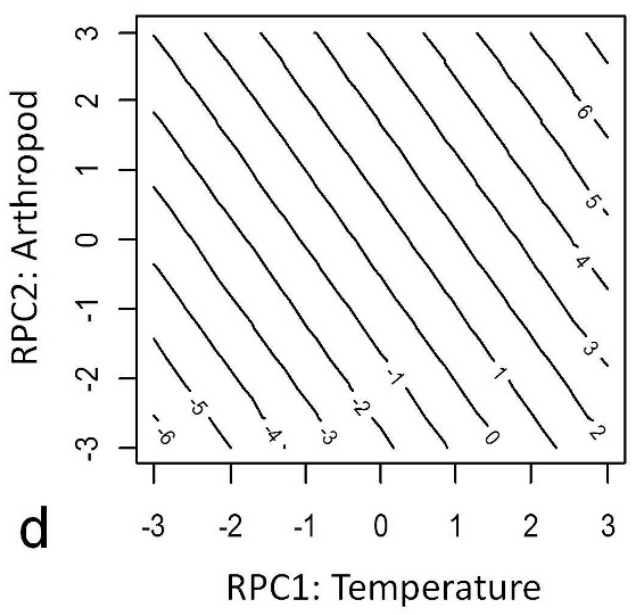

Figure $2 \mid$ Theoretical contour plot (a) with data points graphed across the range of values for temperature and arthropod biomass indices. The actual contour plots of growth residuals for mass (b), tarsus (c) and culmen (d) for individuals greater than 5 days old. On the contour plot, positive signs indicate positive growth residuals (above average growth) and negative signs indicate negative growth residuals (below average growth). The contour plot of mass, tarsus and culmen growth residuals after age 5 indicated that at the mean value of our temperature index, average or above average mass is maintained only in the presence of above average arthropod biomass index values. However, if temperatures increase above the current average, then average or above average mass is maintained even in the presence of the lowest values of the index of arthropod biomass.

thermoregulation immediately upon hatch, and are brooded by parents during the period immediately following hatch ${ }^{17}$, we also included models where both temperature and arthropod biomass interacted with different age classes and tested for random effects of year and family (Supplementary Table S1).

\section{Results}

We found that for all three morphometric measures, variation in growth residuals of known age chicks was best described by a model including additive effects of an index of temperature and an index of arthropod biomass as well as interactive effects of these two indices with a categorical age variable separating young (up to 5 days old) chicks from older ( $>5$ days old) chicks (including a random effect of family; Figure 3, Supplementary Table S2, S3). For chicks 5 days and younger, there were no detectable effects of temperature or arthropod biomass on growth residuals for any of the morphometric measures (Figure 3, Supplementary Table S3). After the age of 5 days, growth residuals increased as indices of temperature and arthropod biomass increased (Figure 3, Supplementary Table S3). As predicted, the positive effect of temperature on chicks over 5 days old was strong enough such that when the index of arthropod biomass was lower than average, above average growth residuals could still be maintained in the presence of increasing temperatures (Figure 2) suggesting that chicks have the potential for physiological relief from the trophic constraints hypothesized by current climate change studies.

\section{Discussion}

By generating independent indices of climate and resource availability and quantifying their effects on chick growth in the sub-arctic tundra, we show that when resource availability is below average, above average growth can still be maintained in the presence of above average temperatures. These results provide evidence that chicks may find physiological relief from the trophic constraints hypothesized by climate change studies. That we detected an interactive effect of age with both of our climate and food availability indices provides evidence of the importance of parental care, specifically brooding behaviour, during the first few days following hatch. Though laboratory studies have indicated that younger chicks may be highly vulnerable to impacts of temperature and food availability ${ }^{19}$, our results indicate that the behaviour of brooding adults appears to diminish this impact in the wild, at least for chicks less than 5 days old. As adults tend to brood young for longer periods of time as ambient 


\section{Age: Less than 5 days}

a

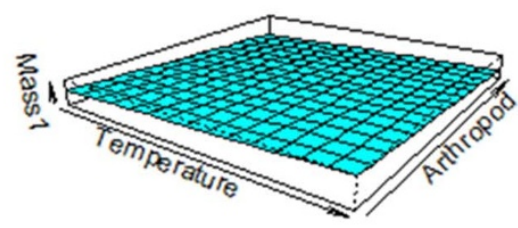

b
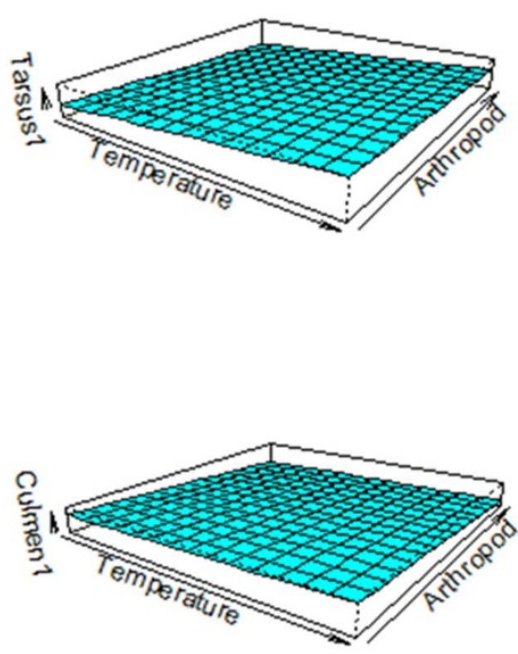

Age: Greater than 5 days
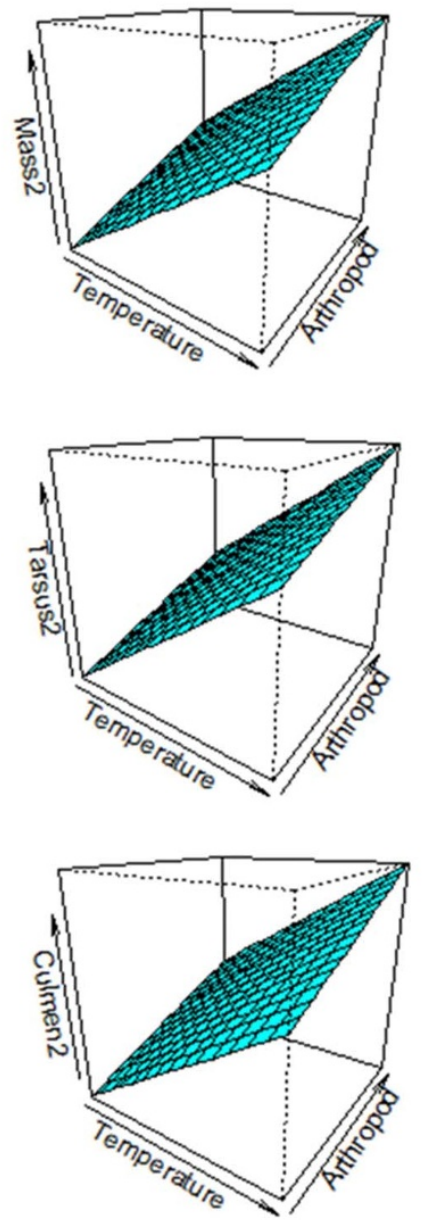

Figure 3 Graphical presentation of the Rpc1*AgeClass_5d interaction and Rpc2*AgeClass_5d interaction for mass by age (a), tarsus by age b) and culmen by age (c) (Supplementary Table S1, S2, bivariate figures available in Supplementary Figure S1). Adding year as random did not improve model fit. All models included family as a random factor, as adding this random component to the most complex model in the first stage of model selection for all measurements improved model fit by at least 67 BIC. Upon comparison of the 15 candidate models, the model including interactions of RPC1*AgeClass_5d and RPC2*AgeClass_5d was by far the best fitting model for all three measures (Supplementary Table S2). Graphical interpretation of this interaction model provided evidence that there were no detectable effects of climate or food availability before age 5 days, however after age 5 days, mass growth residuals increased with higher temperatures and higher arthropod biomass.

temperatures decrease ${ }^{22}$, increases in temperature during the brood rearing season could also have the potential to alleviate costs of parental care, especially for uniparental species for which these costs are particularly high ${ }^{23}$.

Identifying the potentially positive effects of increasing temperatures on reproduction of arctic nesting birds is not in itself novel ${ }^{20,24}$, however, studies investigating the mismatch hypothesis in birds often fail to consider the potential compensatory nature of these positive effects ${ }^{8-11}$. This is likely due to the tight relationship between climatic variables and food availability and the difficulty in teasing apart their influence on growth. In our study the generation of independent indices using principal components analysis on the combined set of climate and food availability variables enabled us to account for multicollinearity between these variables and tease apart their respective effects with greater confidence. Although the principal components analysis does account for the problem of multicollinearity, due to the inextricable physiological link between the climate and arthropod biomass ${ }^{25}$, there is still some influence (loadings) of food variables within our index of climate. It is also important to note that these indices are based on current relationships between variables of climate and food availability at one study site in the low Arctic, therefore, the generality of these results will depend on whether these relationships hold across different habitats and future climate scenarios. Across a large geographical range in the Canadian Arctic where average summer temperatures varied by up to $6.8^{\circ} \mathrm{C}$, relationships between climatic variables such as temperature, wind and rain, and arctic arthropod biomass have been shown to be relatively consistent ${ }^{25}$, providing some support for the generality of our results.

These findings suggest that there may be some positive effects of increasing temperatures on energy requirements and growth in shorebird chicks, and that these effects could compensate for decreases in food availability hypothesized by mismatch studies. Potential decreases in thermoregulatory costs of chicks leading to reduced brooding requirements may also alleviate parent-offspring conflict during the brood-rearing period. Of course, a comprehensive understanding of these bioenergetics trade-offs will require more detailed knowledge of metabolic energy expenditure and how this expenditure changes with the environment ${ }^{26}$. Though data exist on energy expenditure of arctic-nesting shorebirds and their chicks, 
effects of ambient temperature on energetics of free living chicks have not been well studied to date $e^{27,28}$. Given the suite of other potential climate related pressures on arctic-nesting shorebirds (habitat loss due to increased shrub cover and decreases in permafrost, increased predation risk etc. ${ }^{24}$ ), it is now critical to identify whether arcticnesting shorebirds possess the behavioural and physiological mechanisms necessary to mitigate a potential mismatch between hatch and food resources. Understanding behavioural and physiological responses to varying food resources will be key to elucidating whether arctic-nesting species have the capacity to adapt to climateinduced changes in the availability of food resources.

\section{Methods}

This study was conducted within the Churchill Wildlife Management Area in Churchill, Manitoba, Canada $\left(58^{\circ} 45^{\prime} \mathrm{N}, 94^{\circ} 04^{\prime} \mathrm{W}\right)$ located at the eco-zone at the southern limit of sub-arctic tundra and the northern limit of the boreal forest treeline (see ${ }^{29}$ for a detailed physiography of the region). The study area encompassed $\sim 2 \mathrm{~km}^{2}$ of a large open wet sedge meadow environment bordered by dry spruce forest. During the summers of 2010 and 2011, we actively searched for, and subsequently monitored nests of Dunlin, between 1 June and 22 July of each year. Nests found opportunistically outside the main study area were also monitored when possible. For each nest found, information on egg measurements, number of eggs and/or young upon hatch, and incubation stage was collected. Hatch dates were based on confirmed sightings of newly hatched chicks in the nest. When hatch dates were not confirmed by chicks in the nests, hatch dates were estimated based on either 1) known laying dates

(adding 22 days incubation + \# eggs laid), 2) egg flotation ${ }^{30}$, or 3) signs of hatch in the nest (starred or pipped eggs).

All 4 Dunlin eggs generally hatch within 24 hours and chicks often leave the nest within 24 hours after hatch. Chicks that were completely out of the egg were banded with a metal band and an individual combination of 4 colour bands and then weighed to the nearest $0.5 \mathrm{~g}$ with a $30 \mathrm{~g}$ or $100 \mathrm{~g}$ pesola scale. Culmen and tarsus (diagonal) were also measured to the nearest $100^{\text {th }}$ of a millimeter using an electronic caliper. In 2010, observers returned to the previous site where chicks were captured approximately every 2 days and searched for marked broods within a few hundred meters in order to recapture known age chicks. In 2011, chicks were located more systematically by having three observers walk 27-900 m perpendicular transect lines located approximately 50 meters apart within a smaller area of $1 \mathrm{~km}^{2}$ of high Dunlin nesting density. Transects were walked every 2 days and broods located outside the transect area were visited on alternate days. In both years, chicks were re-located by finding banded adults performing distraction displays. When relocated, we re-captured chicks by hand and weighed and re-measured their culmen and tarsus.

We generated growth curves separately for each of the three morphometric measures collected. For mass, culmen and tarsus, we modeled growth of known age birds using 4 different 4-parameter growth models (logistic, Log-logistic, Weibull Type 1, Weibull Type 2) using the package ' $\mathrm{drc}^{331}$ in R v.2.14.1. We did not constrain the upper limit of the models to the average adult size because the age at which captures ceased was generally the age at which chicks were capable of flight (and therefore could not be easily captured). Therefore our growth models provide the empirical asymptote of each measure for newly fledged chicks, not adult-sized young. For each morphometric measure, the model with the lowest AIC score among the 4 models compared was considered the best fitting model, and models with less than $2 \Delta$ AIC from the top model were considered competitive ${ }^{32}$. Any $\Delta$ AIC scores reported refer to the difference in AIC values between the candidate model and the best fitting model.

Climate covariates for the Churchill area were obtained from the National Climate Data and Information Archive, Environment Canada website (http://www.climate. weatheroffice.ec.gc.ca). Variables included minimum, mean and maximum daily temperature $\left({ }^{\circ} \mathrm{C}\right)$, total daily precipitation $(\mathrm{mm})$ and maximum wind speed $(\mathrm{km} / \mathrm{h}$; Supplementary Figure S2). Previous studies have confirmed that each of these variables may be important determinants of growth in arctic-nesting shorebird chicks ${ }^{4,19}$ For each morphometric measurement, a mean for each covariate was calculated for the period from the day before hatch to the day before capture. For individuals measured at hatch, the daily mean of the previous day was used as the hatchling could have been exposed for up to 24 hours. Food availability covariates were obtained by sampling arthropod abundance every 3 days between 23 June and 21 July, using modified pitfall traps (Supplementary Figure S3). Traps were composed of a $38 \mathrm{~cm} \times 5 \mathrm{~cm} \times 7 \mathrm{~cm}$ plastic pitfall trap from which extended a $40 \mathrm{~cm} \times 40 \mathrm{~cm}$ vertical mesh screen to capture both ground dwelling and low flying arthropods. Five traps were placed at $20 \mathrm{~m}$ intervals within an area of high shorebird nesting density. The contents of all traps were sorted and identified to family and sorted into size classes $(<2.9,3-4.9,5-7.9,8-10.9,11-14.9,15-19.9,20-29.9$ and $>30 \mathrm{~mm})$. The midpoint of each size class was used as the length to calculate biomass based on published ${ }^{13,33}$ and unpublished length to biomass equations. When length to biomass equations were not available for identified families, biomass was calculated at the level of order. To account for variable sampling effort, total arthropod biomass was divided by the number of traps sampled, and is presented as arthropod biomass (mg/trap/day). For each morphometric measurement, the mean daily arthropod biomass (mg/trap/day) was calculated for the period from the day before hatch to the day before capture. For individuals measured at hatch, the mean arthropod biomass of the previous day was used.

Previous stomach content analysis of Churchill Dunlin ${ }^{34}$ indicated that diet included, in order of importance, larvae of Tipulidae, plant seeds, larvae of Chironomidae, Dolichopodidae, larvae of miscellaneous Tipulidae, Ephydridae, Gyrinidae, Syrphidae, Trichoptera and Homoptera, larvae of Chrysomelidae, and unidentified snails. Other items found in smaller proportions in the stomach included Dipteran eggs, Dytiscidae larvae, adult Tipulidae, unidentified Coleoptera and adult Dytiscidae $^{34}$. Araneae (spiders), Carabidae, Chironomidae and Tipulidae have also been confirmed in the diet of Dunlin in Alaska ${ }^{35}$. Though the diet of adults are composed of both larval and adult stages, young sandpiper chicks generally forage by catching surface active prey ${ }^{36}$, therefore the surface active prey captured in our modified pitfall traps likely encompass the range of food items available to foraging chicks. Based on these data, we calculated the mean daily arthropod biomass for all arthropod families combined as well as separately for each of the following confirmed prey groups; Araneae, Coloeptera, Diptera, and Trichoptera.

To determine the relative importance of our covariates in explaining variation in growth, we first generated indices of relative growth by extracting the growth residuals (observed - expected) from the best fitting growth model for each morphometric measure. We then constructed linear mixed effects models to explain variation in our indices of growth based on factors related to climate and food availability using the package ' $1 \mathrm{me}{ }^{37}$ in R. v. 2.14.1. We ran a principal components analysis on the 10 variables ( 5 climate and 5 food availability) in order to generate a set of synthetic orthogonal variables to represent climate and food availability independently ${ }^{20}$ using the package 'psych' in R. v. 2.14.1 $1^{38}$. All axes with eigenvalues greater than 1 were retained and a varimax rotation was applied to these axes to improve biological interpretation ${ }^{20}$ (Supplementary Table S6). We then generated a list of 15 a priori defined models based on a combination of additive and interaction effects involving our new synthetic factors, age classes, and two random factors, year and family (Supplementary Table S1). In the 4 cases where chicks from different broods shared at least 1 parent between years, these chicks were assigned the same family identity. Given that shorebird chicks do not gain thermoregulatory independence immediately upon hatch, and are brooded by parents during the period immediately following hatch ${ }^{17}$, we also included models where both temperature and arthropod biomass interacted with different age classes (Hypotheses 14 and 15, Supplementary Table S1). For the linear mixed models, we selected the best fitting model using Bayesian Information Criterion (BIC) as this is considered a more conservative information criterion (higher penalty on models with more parameters) for use with mixed models ${ }^{39}$. The model with the lowest BIC score among the 15 models compared was considered the best fitting model. Any $\triangle \mathrm{BIC}$ scores reported refer to the difference in BIC values between the candidate model and the best fitting model. For the linear mixed models, model selection occurred in three steps ${ }^{39}$. First, the inclusion of random effects was determined by comparing the most complex model (Hypothesis 14 and 15, equal number of parameters) with and without each of the random effects, using the Restricted Maximum Likelihood Estimation (REML) method. The random effects retained in the best fitting model during the first step were then included in each of the 15 a priori models compared in a second model selection step using the Maximum Likelihood (ML) method. The best model was then refitted using the REML method and validated graphically by assessing the spread of the residuals versus fitted values and normality of the residuals. In the event that models with interaction were selected as the best model, graphical interpretation of the interactions was required to determine whether the model supported its associated hypothesis.

All methods in this study were reviewed and accepted by the Animal Care Committee of Trent University. All graphics and statistical analyses were conducted in $\mathrm{zR}$ v.2.14.1.

1. Henningsson, S. S. \& Alerstam, T. Patterns and determinants of shorebird species richness in the circumpolar Arctic. J. Biogeogr. 32, 383-396 (2005).

2. Piersma, T. et al. High daily energy expenditure of incubating shorebirds on High Arctic tundra: a circumpolar study. Funct. Ecol. 17, 356-362 (2003).

3. Miller, E. H. Egg size in the Least Sandpiper Calidris minutilla on Sable Island, Nova Scotia, Canada. Ornis Scand. 10, 10-16 (1979).

4. Schekkerman, H., Tulp, I., Piersma, T. \& Visser, G. H. Mechanisms promoting higher growth rate in arctic than in temperate shorebirds. Oecologia 134, 332-342 (2003).

5. Tjorve, K. M. C., Garcia-Pena, G. E. \& Szekely, T. Chick growth rates in Charadriiformes: comparative analyses of breeding climate, development mode and parental care. J. Avian Biol. 40, 553-558 (2009).

6. O'Reilly, K. M. \& Wingfield, J. C. Seasonal, age, and sex differences in weight, fat reserves, and plasma corticosterone in Western Sandpipers. Condor 105, 13-26 (2003).

7. Visser, M. E., Holleman, L. J. M. \& Gienapp, P. Shifts in caterpillar biomass phenology due to climate change and its impact on the breeding biology of an insectivorous bird. Oecologia 147, 164-172 (2006).

8. Both, C., Bouwhuis, S., Lessells, C. M. \& Visser, M. E. Climate change and population declines in a long-distance migratory bird. Nature 441, 81-83 (2006).

9. Drever, M. C. et al. Population vulnerability to climate change linked to timing of breeding in boreal ducks. Global Change Biol. 18, 480-492 (2012).

10. Both, C. \& Visser, M. E. Adjustment to climate change is constrained by arrival date in a long-distance migrant bird. Nature 411, 296-298 (2001). 
11. Saino, N. et al. Climate warming, ecological mismatch at arrival and population decline in migratory birds. Pro. Roy. Soc. B-Biol. Sci. 278, 835-842 (2011).

12. Gaston, A. J. \& Gilchrist, H. G., Mallory, M. L. \& Smith, P. A. Changes in seasonal events, peak food availability and consequent breeding adjustment in a marine bird: a case of progressive mismatching. Condor 111, 111-119 (2009).

13. McKinnon, L., Picotin, M., Bolduc, E., Juillet, C. \& Bêty, J. Timing of breeding, peak food availability and effects of a mismatch on chick growth in High-Arcticnesting birds. Can. J. Zool. 90, 961-971 (2012).

14. Tulp, I. \& Schekkerman, H. Has prey availability for arctic birds advanced with climate change? Hindcasting the abundance of tundra arthropods using weather and seasonal variation. Arctic 61, 48-60 (2008).

15. Pearce-Higgins, J. W., Dennis, P., Whittingham, M. J. \& Yalden, D. W. Impacts of climate on prey abundance account for fluctuations in a population of a northern wader at the southern edge of its range. Global Change Biol. 16, 12-23 (2010).

16. Schekkerman, H. \& Visser, G. H. Prefledging energy requirements in shorebirds: energetic implications of self-feeding precocial development. Auk 118, 944-957 (2001).

17. Beintema, A. \& Visser, G. Growth parameters in chicks of charadriiform birds Ardea 77, 169-180 (1989).

18. Schekkerman, H., Van Roomen, M. J. W. \& Underhill, L. G. Growth, behaviour of broods and weather-related variation in breeding productivity of Curlew Sandpipers Calidris ferruginea. Ardea 86, 153-168 (1998).

19. Bakken, G. S., Williams, J. B. \& Ricklefs, R. E. Metabolic response to wind of downy chicks of Arctic-breeding shorebirds (Scolopacidae). J. Exp. Biol. 205 2435-3443 (2002).

20. Juillet, C., Choquet, R., Gauthier, G., Lefebvre, J. \& Pradel, R. Carry-over effects of spring hunt and climate on recruitment to the natal colony in a migratory species. J. Appl. Ecol, 49, 1237-1246 (2012).

21. Jolliffe, I. T. Eds. Principal Components Analysis (Springer Series in Statistics, Springer, New York, 2002).

22. Blanken, M. S. \& Nol, E. Factors affecting parental behavior in Semipalmated Plovers. Auk 115, 166-174 (1998).

23. Tulp, I. et al. Energetic demands during incubation and chick rearing in a uniparental and biparental shorebird breeding in the High Arctic. Auk 126, 155-164 (2009).

24. Meltofte, H. et al. Effects of climate variation on the breeding ecology of Arctic shorebirds. Bioscience 59, 1-48 (2007).

25. Bolduc, E. et al. Terrestrial arthropod abundance and phenology in the Canadian Arctic: modeling resource availability for arctic-nesting insecivorous birds. Can. Entomol. 145, 1-16 (2013).

26. Humphries, H. H., Thomas, D. W. \& Speakman, J. R. Climate-mediated energetic constraints on the distribution of hibernating mammals. Nature 418, 313-316 (2002).

27. Williams, J. B., Tieleman, B. I., Visser, G. H. \& Ricklefs, R. E. Does growth rate determine the rate of metabolism in shorebird chicks living in the arctic? Physiol. Biochem. Zool. 80, 500-513 (2007).

28. Krijgsveld, K. L., Ricklefs, R. E. \& Visser, G. H. Daily energy expenditure in precocial shorebird chicks: smaller species perform at higher levels. J. Ornithol 153, 1203-1214 (2012)

29. Scott, P. A. \& Stirling, I. Chronology of terrestrial den use by polar bears in western Hudson Bay as indicated by tree growth anomalies. Arctic 55, 151-166 (2002).
30. Liebezeit, J. R. et al. Assessing the development of shorebird eggs using the flotation method: Species-specific and generalized regression models. Condor 109, 32-47 (2007).

31. Ritz, C. \& Streibig, J. C. Bioassay Analysis using R. J. Statist. Software 12 (2005).

32. Burnham, K. P. \& Anderson, D. R. Model Selection and Multimodel Inference: A Practical Information-Theoretic Approach. (Springer-Verlag, 2002).

33. Sample, B. E., Cooper, R. J., Greer, R. D. \& Whitmore, R. C. Estimation of insect biomass by length and width. Am. Midl. Nat. 129, 234-240 (1993).

34. Baker, M. C. Shorebird food habits in the eastern Canadian Arctic. Condor 79, 56-62 (1977)

35. Holmes, R. T. \& Pitelka, F. A. Food overlap among coexisting sandpipers on northern alaskan tundra. Syst. Zool. 17, 305-318 (1968).

36. Nice, M. M. Development of behavior in precocial birds. Trans. Linn. Soc. N. Y. 8 (1962).

37. Pinheiro, J., Bates, D., DebRoy, S., Sarkar, D. \& Team, R. D. C. nlme: Linear and Nonlinear Mixed Effects Models. (R package version 3-103, 2012).

38. Revelle, W. psych: Procedures for personality and psychological research ( $\mathrm{R}$ package version 1.1.12, 2011).

39. Zurr, A. F., Ieno, E. N., Walker, N. J., Saveliev, A. A. \& Smith, G. M. Mixed Effects Models and Extensions in Ecology with R. (Springer, 2009).

\section{Acknowledgements}

This study was made possible due to funding by a Natural Sciences and Engineering Research Council of Canada (NSERC) Postdoctoral Fellowship to LM, an NSERC Discovery Grant and Northern Research Supplement to EN, the Symons Trust Fund for Canadian Studies, the Churchill Northern Studies Centre Research Fund, the Northern Scientific Training Program, Manomet Centre for Conservation Sciences funding via the Arctic Shorebird Demographic Network and Environment Canada Science Horizons Youth Internship program. We thank L. Fishback and R. Gates for logistical support in the field. Thanks are due to the field assistants who collected arthropods and chick growth data in the field; E. D'Astous, J-R. Julien, F. Rousseu and C. Lishman, and the technicians who identified arthropods in the laboratory; D. Turner and L. Pollock.

\section{Author contributions}

L.M. designed the study and collected and analysed the data. L.M., E.N. and C.J. interpreted the results and wrote the manuscript together.

\section{Additional information}

Supplementary information accompanies this paper at http://www.nature.com/ scientificreports

Competing financial interests: The authors declare no competing financial interests.

License: This work is licensed under a Creative Commons

Attribution-NonCommercial-NoDerivs 3.0 Unported License. To view a copy of this license, visit http://creativecommons.org/licenses/by-nc-nd/3.0/

How to cite this article: McKinnon, L., Nol, E. \& Juillet, C. Arctic-nesting birds find physiological relief in the face of trophic constraints. Sci. Rep. 3, 1816; DOI:10.1038/ srep01816 (2013). 